\title{
LARGE-TIME BEHAVIOR OF SOLUTIONS TO CERTAIN QUASILINEAR PARABOLIC EQUATIONS IN SEVERAL SPACE DIMENSIONS
}

\author{
PATRICIA BAUMAN AND DANIEL PHILLIPS ${ }^{1}$
}

\begin{abstract}
We consider the Cauchy problem, $u_{t}+\operatorname{div} f(u)=\Delta u$ for $x \in \mathbf{R}^{n}, t>0$ with $u(x, 0)=u_{0}(x)$. For $n=1$, suppose $f^{\prime \prime}>0$ and $\int\left|u_{0}-\phi\right| d x<\infty$ where $\phi$ is piecewise constant and $\phi(x) \rightarrow u^{+}\left(u^{-}\right)$as $x \rightarrow+\infty(-\infty)$. A result of Il'in and Oleinik states that if $\phi(x-k t)$ is an entropy solution of $u_{t}+\operatorname{div} f(u)=0$, then $u(x, t)$ approaches a traveling wave solution, $\tilde{u}(x-k t)$, as $t \rightarrow \infty$, with $\tilde{u}(x) \rightarrow u^{+}$ $\left(u^{-}\right)$as $x \rightarrow+\infty(-\infty)$. We give two examples which show that this result does not hold for $n \geqslant 2$.
\end{abstract}

This work concerns the asymptotic behavior of the solution to the Cauchy problem,

$$
\begin{aligned}
& u_{t}+\operatorname{div} f(u)=\Delta u \text { for } x \in \mathbf{R}^{n}, t>0, \\
& u(x, 0)=u_{0}(x) \text { for } x \in \mathbf{R}^{n},
\end{aligned}
$$

where $f \in C^{2}\left(\mathbf{R} ; \mathbf{R}^{n}\right)$, and $n \geqslant 2$. We assume throughout the paper that $u_{0}$ is a bounded function which approaches a piecewise constant state as $|x| \rightarrow \infty$ in the sense that $\int_{\mathbf{R}^{\prime \prime}}\left|u_{0}-\phi\right| d x<\infty$, where

$$
\phi(x)=a \cdot \chi_{\Omega}(x)+b \cdot \chi_{\mathbf{R}^{\prime \prime} \backslash \Omega}(x)
$$

with $a \geqslant b$, and $\Omega$ is a connected open subset of $\mathbf{R}^{n}$ (with piecewise smooth boundary if $n \geqslant 2$ ). We also assume without loss of generality that $f(a)=f(b)=0$, since the transformation $\bar{x}=x-k t, \bar{t}=t$, where $k=[f(a)-f(b)] /(a-b)$, yields an equation of the form (1) which satisfies this condition.

For $n=1$, a result of Il'in and Oleinik [3] states that if $f$ is strictly convex and

$$
\phi(x)=\phi_{c}(x) \equiv \begin{cases}a, & x<c \\ b, & x>c,\end{cases}
$$

for some $c \in \mathbf{R}$, then $\lim _{t \rightarrow \infty} u(x, t) \equiv \tilde{u}(x)$ exists and satisfies

$$
\begin{aligned}
& \operatorname{div} f(\tilde{u})=\Delta \tilde{u} \quad \text { in } \mathbf{R}^{n}, \\
& \tilde{u}(x)-\phi(x) \rightarrow 0 \quad \text { as }|x| \rightarrow \infty, \text { and } \\
& \int_{\mathbf{R}^{n}}(\tilde{u}-\phi) d x=\int_{\mathbf{R}^{n}}\left(u_{0}-\phi\right) d x .
\end{aligned}
$$

Received by the editors February 4, 1985.

1980 Mathematics Subject Classification. Primary 35L65; Secondary 35K55.

${ }^{1}$ Supported by the National Science Foundation, Grant No. 8201036.

C1986 American Mathematical Society $0002-9939 / 86 \$ 1.00+\$ .25$ per page 
Note that any function $\phi$ of the form (3) satisfies

$$
u_{t}+\operatorname{div} f(u)=0 \text {. }
$$

Also since $f$ is convex, the functions $\left\{\phi_{c}\right\}$ are precisely those of the form (3) which satisfy the entropy condition (see (E) below). Thus in one space dimension, $\lim _{t \rightarrow \infty} u(x, t)$ exists and satisfies (4) and (5) whenever $\phi$ satisfies (E).

It is easy to see that the converse is also true. That is, if $\lim _{t \rightarrow \infty} u(x, t)$ exists and satisfies (4) and (5), there is a function $\psi$ of the form (3) which satisfies the entropy condition and

$$
\int_{\mathbf{R}}|\phi-\psi| d x<\infty .
$$

The entropy condition for solutions of (6) when $n \geqslant 1$ was formulated by Kruzkov and Vol'pert in [4 and 6]. When applied to $\phi$ as in (3), it can be stated as follows:

$$
\langle f(c), n(x)\rangle \leqslant 0 \text { for all } c+(b, a)
$$

$H^{n-1}$-almost everywhere on $\partial \Omega$, where $n(x)$ is the outward-pointing normal to $\Omega$ at $x$.

In this paper we give two examples which demonstrate that Il'in and Oleinik's result on the large-time behavior of solutions to (1) and (2) fails in dimension $n \geqslant 2$. In both examples, $f$ satisfies the strong convexity condition formulated by Conway in [1]. (In fact, we take $f=(0, \ldots, 0, F)$ with $F$ strictly convex.) We take $u_{0}=\phi$ where $\phi$ has the form (3) and $\phi$ satisfies (E).

In our first example, $\tilde{u}(x) \equiv \lim _{t \rightarrow \infty} u(x, t)$ exists and satisfies the elliptic equation (4), but does not inherit the asymptotic values of $\phi$ as $|x| \rightarrow \infty$, as in (5). In our second example, $\tilde{u}$ fails to exist.

EXAMPLE 1. Let $f(u)=(0,0, \ldots, F(u))$ where $F(u)$ is a smooth strictly convex function with $F(0)=F(1)=0$. Set $\phi(x)=\chi_{\Omega}(x)$ where

$$
\Omega=\left\{x=\left(x^{\prime}, x_{n}\right): x_{n}<-\left|x^{\prime}\right|\right\} .
$$

One readily checks that $\phi$ satisfies condition (E).

We take $u(x, t)$ to be the solution of (1) and (2) with $u_{0}(x)=\phi(x)$. Such a solution will exist and be unique in the class

$$
C\left([0, \infty) ; L_{\mathrm{loc}}^{1}\left(\mathbf{R}^{n}\right)\right) \cap L^{\infty}\left(\mathbf{R}^{n+1}\right) \cap C^{2}\left(\mathbf{R}^{n} \times(0, \infty)\right)
$$

and satisfies $0 \leqslant u \leqslant 1$. This can be seen by considering a sequence of smooth, bounded functions $u_{0}^{N}(x) \rightarrow \phi(x)$ in $L_{\text {loc }}^{1}\left(\mathbf{R}^{n}\right)$. Taking the corresponding solutions of the Cauchy problem [5, V, Theorem 8.1] and arguing as in [6, §17.2], one obtains both existence and uniqueness in the indicated class. We will show that $\lim _{t \rightarrow \infty} u(x, t) \equiv 0$.

Consider first for the sake of comparison the function $g_{d}\left(x_{n}, t\right)$ satisfying

$$
\begin{gathered}
D_{x_{n} x_{n}} g_{d}=D_{t} g_{d}+D_{x_{n}}\left(F\left(g_{d}\right)\right) \text { for }-\infty<x_{n}<\infty, t>0, \\
g_{d}\left(x_{n}, 0\right)= \begin{cases}1, & x_{n}<d, \\
0, & x_{n}>d .\end{cases}
\end{gathered}
$$


From [3] the functions

$$
\int_{-\infty}^{-y}\left(1-g_{d}(s, t)\right) d s, \quad \int_{y}^{\infty} g_{d}(s, t) d s
$$

exist, are continuous for $y \in \mathbf{R}$ and $t \geqslant 0$, and together with

$$
\left(1-g_{d}(-y, t)\right), \quad g_{d}(y, t)
$$

tend to zero as $y \rightarrow+\infty$ uniformly in $t$.

Set $V_{d}\left(x_{n}, t\right)=\int_{x_{n}}^{\infty} g_{d}(s, t) d s$; this is continuous for $t \geqslant 0$ and satisfies

$$
D_{x_{n} x_{n}} V_{d}=-F\left(-D_{x_{n}} V_{d}\right)+D_{t} V_{d}, \quad V_{d}\left(x_{n}, 0\right)=\left(d-x_{n}\right)^{+} \text {. }
$$

Moreover

$$
\tilde{g}_{d}\left(x_{n}\right) \equiv \lim _{t \rightarrow \infty} g_{d}\left(x_{n}, t\right) \quad \text { and } \quad \tilde{V}_{d}\left(x_{n}\right) \equiv \lim _{t \rightarrow \infty} V_{d}\left(x_{n}, t\right)
$$

both exist and are solutions to (7) and (8); they are uniquely determined respectively by

$$
\int_{d}^{\infty} \tilde{g}_{d}(s) d s+\int_{-\infty}^{d}\left(\tilde{g}_{d}(s)-1\right) d s=0, \quad \text { and } \quad \tilde{V}_{d}\left(x_{n}\right)=\int_{x_{n}}^{\infty} \tilde{g}_{d}(s) d s .
$$

Finally the convergence to $\tilde{g}_{d}$ is uniform in $x_{n}$.

From the maximum principle

$$
0 \leqslant u(x, t) \leqslant g_{0}\left(x_{n}, t\right) \text { for } x \in \mathbf{R}^{n}, t \geqslant 0 .
$$

Thus $U(x, t)=\int_{x_{n}}^{\infty} u\left(x^{\prime}, s, t\right) d s$ is well defined and satisfies

$$
\Delta U=-F\left(-D_{x_{n}} U\right)+D_{t} U, \quad U(x, 0)=\left(-\left|x^{\prime}\right|-x_{n}\right)^{+} .
$$

For any $d<0, w_{d} \equiv U-V_{d}$ satisfies

$$
\Delta w_{d}=a(x, t, d) D_{x_{n}} w_{d}+D_{t} w_{d} \quad \text { and } \quad w_{d}(x, 0) \leqslant-d \cdot \chi_{\left\{\left|x^{\prime}\right| \leqslant-d\right\}}(x) .
$$

Again using the maximum principle, $w_{d} \leqslant h\left(x^{\prime}, t\right)$ for $t \geqslant 0$ where

$$
\Delta_{x^{\prime}} h=D_{t} h \text { for } x^{\prime} \in \mathbf{R}^{n-1}, t>0
$$

and

$$
h\left(x^{\prime}, 0\right)=-d \cdot \chi_{\left\{\left|x^{\prime}\right| \leqslant-d\right\}}\left(x^{\prime}\right), \quad x^{\prime} \in R^{n-1} .
$$

Hence

$$
\varlimsup_{t \rightarrow \infty} U(x, t) \leqslant \tilde{V}_{d}\left(x_{n}\right)+\lim _{t \rightarrow \infty} h\left(x^{\prime}, t\right)=\tilde{V}_{d}\left(x_{n}\right) .
$$

Since $g_{d}\left(x_{n}, t\right)=g_{0}\left(x_{n}-d, t\right), \tilde{V}_{d}\left(x_{n}\right) \rightarrow 0$ as $d \rightarrow-\infty$. From parabolic estimates it follows that

$$
u(x, t)=-D_{x_{n}} U(x, t) \rightarrow 0 \text { as } t \rightarrow \infty .
$$

EXAmple 2. Consider $f(u)$ as above and $u_{0}(x)=\phi(x)=\chi_{\Omega}(x)$ where

$$
\Omega=\left\{\left(x^{\prime}, x_{n}\right): x_{n}<\psi\left(x^{\prime}\right)\right\} \quad \text { with } \psi \in C^{1}\left(\mathbf{R}^{n-1}\right),-1 \leqslant \psi \leqslant 0,
$$

and

$$
\varlimsup_{r \rightarrow \infty} r^{1-n} \int_{\left|x^{\prime}\right| \leqslant r} \psi d x^{\prime} \neq \underset{r \rightarrow \infty}{\lim } \int_{\left|x^{\prime}\right| \leqslant r} \psi d x^{\prime}
$$


Again one can check that (E) is satisfied. We show that $\lim _{t \rightarrow \infty} u(x, t)$ cannot exist pointwise almost everywhere on $\mathbf{R}^{n}$.

Using the previous remarks on $g_{d}$ we see that

$$
g_{-1}\left(x_{n}, t\right) \leqslant u(x, t) \leqslant g_{0}\left(x_{n}, t\right)
$$

and that the function

$$
U\left(x^{\prime}, t\right) \equiv \int_{-\infty}^{0}\left(1-u\left(x^{\prime}, s, t\right) d s-\int_{0}^{\infty} u\left(x^{\prime}, s, t\right) d s\right.
$$

is well defined and satisfies

$$
\begin{aligned}
& \Delta_{x} U=D_{t} U \text { for } t>0, x^{\prime} \in \mathbf{R}^{n-1}, \\
& U\left(x^{\prime}, 0\right)=-\psi\left(x^{\prime}\right) \text { for } x^{\prime} \in \mathbf{R}^{n-1} .
\end{aligned}
$$

If $\lim _{t \rightarrow \infty} u(x, t)$ is well defined, then $\lim _{t \rightarrow \infty} U\left(x^{\prime}, t\right) \equiv \tilde{U}\left(x^{\prime}\right)$ exists. Hence $\tilde{U}$ is a bounded harmonic function and thus a constant. By a result of [2] this is true iff

$$
\lim _{r \rightarrow \infty} r^{1-n} \int_{\left|x^{\prime}\right| \leqslant r} \psi d x^{\prime} \text { exists. }
$$

\section{REFERENCES}

1. E. D. Conway, The formation and decay of shocks for a conservation law in several dimensions, Arch. Rational Mech. Anal. 64 (1977), 47-57.

2. S. D. Eidel'man and V. D. Repnikov, A new proof of the theorem on the stabilization of the solution of the Cauchy problem for the heat equation, Mat. Sb. 73 (115) (1967); English transl., Math. USSR-Sb. 2 (1967), 135-139.

3. A. M. Il'in and O. A. Oleinik, Behavior of the solutions of the Cauchy problem for certain quasilinear equations for unbounded increase of the time, Dokl. Akad. Nauk SSSR 120 (1958), 25-28; English transl., Amer. Math. Soc. Transl. (2) 42 (1964), 19-23.

4. S. Kruzkov, First-order quasilinear equations with several space variables, Mat. Sb. 123 (1970), 228-255; English transl., Math. USSR-Sb. 10 (1970), 217-273.

5. O. A. Ladyzenskaja, V. A. Solonnikov and N. N. Ural'ceva, Linear and quasilinear equations of parabolic type, Amer. Math. Soc., Providence, R. I., 1968.

6. A. I. Vol'pert, The spaces BV and quasilinear equations, Mat. Sb. 73 (115) (1967), 255-302; English transl., Math. USSR-Sb. 2 (1967), 225-267.

Department of Mathematics, Purdue University, West Lafayette, Indiana 47907 